\title{
FRACTIONALLY-SPACED CONSTANT MODULUS ALGORITHM BLIND EQUALIZER ERROR SURFACE CHARACTERIZATION: EFFECTS OF SOURCE DISTRIBUTIONS
}

\author{
James P. LeBlanc ${ }^{1}$ \\ Inbar Fijalkow ${ }^{2}$ \\ C. Richard Johnson, $\mathrm{Jr}^{3}{ }^{3}$ \\ ${ }^{1}$ Dept. of Elec. Eng. \\ ${ }^{2}$ ENSEA/ETIS \\ Rochester Institute of Technology \\ 6 avenue du Ponceau \\ 95014 Cergy-Pontoise Cdx, France
${ }^{3}$ School of Elec. Eng.
Cornell University
Ithaca, NY USA

\begin{abstract}
The Constant Modulus Algorithm (CMA) [11], [5] is a popular blind equalization algorithm. A common device used in demonstrating the convergence properties of CMA is the assumption that the source sequence is iid (independent, identically distributed). Recent results in the literature show that a finite length fractionally-spaced equalizer allows for perfect equalization of moving average channels (under certain channel conditions known as zero-forcing criteria). CMA has been shown to converge to such perfectly equalizing settings under an independent, platykurtic source [9], [4]. This paper investigates the effect of the distribution from which an independent source sequence is drawn on the CMA error surface and stationary points in the perfectly-equalizable fractionally-sampled equalizer case. Results include symbolic identification of all stationary points, as well as the eigenvalues and eigenvectors associated with their Hessian matrix. Results show quantitatively the loss of error surface curvature (in both direction and magnitude) at all stationary points. Simulations included demonstrate the affect this has on convergence speed.
\end{abstract}

\section{INTRODUCTION}

This work utilizes the sub-channel (or multivariate) model (Figure 1) of a fractionally sampled CMA blind equalizer in which: sub-channel disparity exists, equalizer time support at least meets that of the channel (as in [10]), and no channel noise. Under such conditions, the combined channelequalizer response $H=\left[\begin{array}{lll}h_{0} & \ldots & h_{n}\end{array}\right]^{t}$ may achieve any parameterization (i.e. all of $H$-space is achievable).

There is a known class of source distributions other than the uniform distribution for which CMA properly behaves (having stability of all perfectly-equalizable parametrizations). This term, in general, for this class is sub-Gaussian [1]. Herein we quantify how the source distribution changes the CMA error surface.

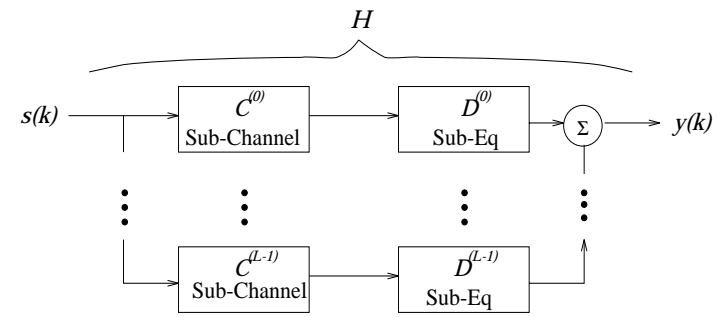

Figure 1. FSE CMA Equalization Block

\footnotetext{
${ }^{1,3}$ Supported in part by Applied Signal Technology, Inc. and NSF Grant MIP-9509011
}

\section{STATIONARY POINTS OF CMA}

With, $F(H)$ written as

$$
\left[\begin{array}{c}
\sum_{i} \mathcal{R}_{i}^{i} h_{i}^{3}+3 \sum_{i \neq j} \mathcal{R}_{i}^{i} h_{i}^{2} h_{j}+\sum_{i \neq j \neq k} \mathcal{R}_{j}^{i} h_{i} h_{j} h_{k}-\gamma \sum_{i} \mathcal{R}_{i}^{0} h_{i} \\
\vdots \\
\sum_{i} \mathcal{R}_{i}^{i} h_{i}^{n}+3 \sum_{i \neq j} \mathcal{R}_{\substack{i \\
i}}^{n} h_{i}^{2} h_{j}+\sum_{i \neq j \neq k} \mathcal{R}_{j}^{i} h_{i} h_{j} h_{k}-\gamma \sum_{i} \mathcal{R}_{i}^{n} h_{i}
\end{array}\right](1)
$$

wherein $\mathcal{R}_{k}^{i}=\mathrm{E}\left\{s_{m-i} s_{m-j} s_{m-k} s_{m-\ell}\right\}$ and $\mathcal{R}_{j}^{i}=$ $\mathrm{E}\left\{s_{m-i} s_{m-j}\right\}$ represent the fourth and second source moments [8], and $\gamma$ is the ratio of the $4^{\text {th }}$ to $2^{\text {nd }}$ moments of the source, the stationary points of CMA are the values of $H$ for which $F(H)=\overrightarrow{0}$. Given that all $H$-space is reachable, the solution to $F(H)=\overrightarrow{0}$ is independent of the channel.

\subsection{Tap Constraints for IID Source}

An important concept in the stability analysis of the CMA stationary points is the dimensionless quantity known as the kurtosis $^{*}$ (see [3]). Denote the central moments of the source by $m_{n}=\mathrm{E}\left\{(s-\mu)^{n}\right\}$ where $\mu=\mathrm{E}\{s\}$. Then kurtosis is defined to be the ratio, $\kappa_{s}=m_{4} / m_{2}^{2}$. The Gaussian distribution has $\kappa_{s}=3$ and is regarded as forming the boundary between platykurtic (low kurtosis distributions, $\kappa_{s}<3$ ) and leptokurtic (high kurtosis distributions, $\left.\kappa_{s}>3\right)$.

For iid sources, all the correlation coefficients of $F(H)$ are zero, except $\mathcal{R}_{i}^{i}{ }_{i}^{i}=\mathrm{E}\left\{s^{4}\right\}, \mathcal{R}_{j}^{i}=\left(\mathrm{E}\left\{s^{2}\right\}\right)^{2}$ for $i \neq j$ and $\mathcal{R}_{i}^{i}=\mathrm{E}\left\{s^{2}\right\}$, then the $n+1$ equations of $F(H)$ are rewritten as $f_{m}, 0 \leq m \leq n$,

$$
f_{m}=m_{2}^{2} h_{m}\left(\kappa_{s} h_{m}^{2}+3 \sum_{i \neq m} h_{i}^{2}-\kappa_{s}\right)
$$

The CMA stationary points are the values of $H$ solving each $f_{m}=0$. It is seen that any $h_{m}=0$ trivially solves $f_{m}=0$. So, only $f_{m}$ for which $h_{m} \neq 0$ need be further considered. Now, for any $h_{m} \neq 0$ we have the condition,

$$
\kappa_{s} h_{m}^{2}+3 \sum_{i \neq m} h_{i}^{2}-\kappa_{s}=0 .
$$

*Some definitions of kurtosis use $\kappa=\frac{m_{4}}{m_{2}^{2}}-3$. 
In the case that $\kappa_{s}=3$, all the $f_{m}$ equations are the same. That is, our system is degenerate and we truly have only one equation to satisfy which has the solution $\sum_{i} h_{i}^{2}=1$. In this case, the stationary points in $H$ are not a discrete set but a dense set corresponding to a unit sphere. Thus, for $\kappa_{s}=3$ CMA adaptation will converge to this sphere and then will wander about the sphere, a generalization of [2].

Alternatively, when $\kappa_{s} \neq 3$ each non-zero $h_{m}$ in a solution set must solve each equation $f_{m}$, we then have that the value of $h_{m}^{2}$ for all non-zero $h_{m}$ in this solution must be the same (i.e. all $h_{m}$ have the same magnitude), a generalization of [6].

\subsection{Classes of Solutions}

For the iid source case, the value of each non-zero $h_{m}$ solving $F(H)=\overrightarrow{0}$ will depend on the total number of non-zero elements of $H$. Divide the set of solutions of into classes, defining the class,

$$
\begin{aligned}
\mathcal{C}_{N}=\{H: F(H) & =\overrightarrow{0} \text { where } H \text { has exactly } \\
& N \text { non-zero elements }\} .
\end{aligned}
$$

Furthermore, denote the size of the class (i.e. the number of its members) by $\left|\mathcal{C}_{N}\right|$. Of the total $n+1$ elements of $H$, since each of its $N$ non-zero elements may have one of two values and there are $\left(\begin{array}{c}n+1 \\ N\end{array}\right)$ ways to group $N$ elements we have,

$$
\left|\mathcal{C}_{N}\right|=2^{N}\left(\begin{array}{c}
n+1 \\
N
\end{array}\right)
$$

Thus, the total number of CMA stationary points under iid source is $3^{n+1}$.

To see that class membership defines the magnitude of the non-zero taps, note that for $H \in \mathcal{C}_{N}$ (3) becomes

$$
\kappa_{s} h_{m}^{2}+3(N-1) h_{i}^{2}-\kappa_{s}=0
$$

yielding

$$
h_{m}=\left\{\begin{array}{l}
0 \\
\pm \sqrt{\frac{\kappa_{s}}{\kappa_{s}-3+3 N}} \quad 0 \leq m \leq n .
\end{array}\right.
$$

\subsection{Categorization of Solutions by Hessian}

Determination of the Hessian of the CMA error surface for the diagonal elements yields

$$
\mathbb{M}(m, m)=\frac{\partial f_{m}}{\partial h_{m}}=m_{2}^{2}\left(3 \kappa_{s} h_{m}^{2}+3 \sum_{i \neq m} h_{i}^{2}-\kappa_{s}\right)
$$

Similarly for the off-diagonal elements $(m \neq n)$,

$$
\mathbb{M}(m, n)=\frac{\partial f_{m}}{\partial h_{n}}=6 m_{2}^{2} h_{n} h_{m}
$$

All the elements of $\mathbb{M}$ evaluated at a stationary point in $\mathcal{C}_{N}$ for $N \geq 1$ contain a strictly positive common factor of $m_{2}^{2}\left(\frac{\kappa_{s}}{\kappa_{s}-3+3 N}\right)$. Factor this out, introducing $\widehat{\mathbb{M}}$ related by,

$$
\mathbb{M}=m_{2}^{2}\left(\frac{\kappa_{s}}{\kappa_{s}-3+3 N}\right) \widehat{\mathbb{M}}
$$

The diagonal elements $\widehat{\mathbb{M}}$ may be described by,

$$
\widehat{\mathbb{M}}(m, m)=\left\{\begin{array}{cc}
3-\kappa_{s}, & h_{m}=0 \\
2 \kappa_{s}, & h_{m} \neq 0 .
\end{array}\right.
$$

Off-diagonal terms are,

$$
\widehat{\mathbb{M}}(m, n)=\left\{\begin{aligned}
6, & h_{m}=h_{n} \neq 0, \\
-6, & h_{m}=-h_{n} \neq 0, \\
0, & \text { else. }
\end{aligned}\right.
$$

The eigenvalues of $\widehat{\mathbb{M}}$ are merely scaled versions of the eigenvalues of $\mathbb{M}$. In this sense, we investigate the eigenvalues of $\widehat{\mathbb{M}}$ to ascertain categorization of a stationary point.

\subsection{Stationary Point Categorization by Class Membership}

For $H \in \mathcal{C}_{0}$ (i.e. the origin), we have $\mathbb{M}=-\kappa_{s} \mathbb{I}$, where $\mathbb{I}$ is the identity matrix. Since $\kappa_{s}$ is strictly positive, $\mathbb{M}$ is negative definite and $H=\overrightarrow{0}$ is a local maximum.

For $H \in \mathcal{C}_{1}$ wherein the solution has a single non-zero tap element, $\widehat{\mathbb{M}}$ is a diagonal matrix with elements given by (11). The diagonal entry corresponding to the single nonzero $h_{m}$ is $2 \kappa_{s}$ (which is strictly positive). The remaining diagonal entries have value $3-\kappa_{s}$. The sign of these entries (and hence, the corresponding eigenvalues) are determined by the kurtosis of the source signal, $\kappa_{s}$. The cases are:

$\boldsymbol{\kappa}_{s}<$ 3: For platykurtic source distributions, all diagonal entries of $\widehat{\mathbb{M}}$ are positive and these channel-equalizers parametrizations are stable (i.e. local minima).

$\boldsymbol{\kappa}_{s}>$ 3: For leptokurtic source distributions, there is the one positive diagonal entry of $2 \kappa_{s}$ and all remaining diagonal entries of $\widehat{\mathbb{M}}$ are $3-\kappa_{s}$ which are negative and these parametrizations are saddle points (i.e. 1 positive eigenvalue, rest negative).

$H \in \mathcal{C}_{N}, \quad 2 \leq N \leq n$, the stationary points are found to be saddle points, regardless of the distribution of the iid source. To see this define the ordered index lists,

$$
\mathcal{I}=\left\{i: h_{i} \neq 0\right\} \quad \text { and } \quad \mathcal{O}=\left\{i: h_{i}=0\right\}
$$

Concatenating these ordered list, we form $\mathcal{I O}$ which lists the ascending indices of the non-zero $h_{m}$ followed by the ascending indices of the zero valued $h_{m}$.

To evaluate the eigenvalues of $\widehat{\mathbb{M}}$ realize that similarity transformations do not change the eigenvalues of a matrix. (If $\mathbb{B}=\mathbb{P}^{-1} \widehat{\mathbb{M} P}$, then $\mathbb{B}$, and $\widehat{\mathbb{M}}$ have the same eigenvalues.) Form the transformation matrix $\mathbb{P}$ as,

$$
\mathbb{P}\left(m, \mathcal{I O}_{m}\right)=\operatorname{sign}\left(h_{\mathcal{I O}_{m}}\right)
$$

and note that $\mathbb{P}$ is unitary $\left(\mathbb{P P}^{t}=\mathbb{I}\right)$.

An example for the case

$$
H^{t}=\sqrt{\frac{\kappa_{s}}{\kappa_{s}+6}}\left[\begin{array}{llllll}
0 & 1 & 1 & 0 & -1 & 0
\end{array}\right]
$$

follows. The index list is $\mathcal{I O}=\{1,2,4,0,3,5\}$. We then write,

$$
\mathbb{B}=\mathbb{P}^{-1} \widehat{M} \mathbb{P}
$$




$$
\begin{aligned}
& {\left[\begin{array}{cccccc}
2 \kappa_{s} & 6 & 6 & & & \\
6 & 2 \kappa_{s} & 6 & & & \\
6 & 6 & 2 \kappa_{s} & & & \\
& & & 3-\kappa_{s} & 0 & 0 \\
& & & 0 & 3-\kappa_{s} & 0 \\
& & & 0 & 0 & 3-\kappa_{s}
\end{array}\right]=} \\
& \mathbb{P}^{-1}\left[\begin{array}{cccccc}
3-\kappa_{s} & 0 & 0 & 0 & 0 & 0 \\
0 & 2 \kappa_{s} & 6 & 0 & -6 & 0 \\
0 & 6 & 2 \kappa_{s} & 0 & -6 & 0 \\
0 & 0 & 0 & 3-\kappa_{s} & 0 & 0 \\
0 & -6 & -6 & 0 & 2 \kappa_{s} & 0 \\
0 & 0 & 0 & 0 & 0 & 3-\kappa_{s}
\end{array}\right] \mathbb{P}
\end{aligned}
$$

where

$$
\mathbb{P}=\left[\begin{array}{cccccc}
0 & 1 & 0 & 0 & 0 & 0 \\
0 & 0 & 1 & 0 & 0 & 0 \\
0 & 0 & 0 & 0 & -1 & 0 \\
1 & 0 & 0 & 0 & 0 & 0 \\
0 & 0 & 0 & 1 & 0 & 0 \\
0 & 0 & 0 & 0 & 0 & 1
\end{array}\right]
$$

The similarity transformation of $\widehat{\mathbb{M}}$ into $\mathbb{B}$ yields a block form matrix, wherein one block is $\left(3-\kappa_{s}\right) \mathbb{I}$ of dimension equal to the number of $h_{m}$ which are zero. The other block is an $N \times N$ circulant matrix with strictly positive elements. Such structure allows easy identification of the eigenvalues. Interestingly, the signs of the eigenvalues of both sub-matrices are determined by the source kurtosis $\kappa_{s}$.

Diagonal Sub-Matrix: The diagonal sub-matrix eigenvalues are $3-\kappa_{s}$. For $\kappa_{s}<3$, this sub-matrix is positive definite. However, for $\kappa_{s}>3$ we have a negative definite sub-matrix. The case $\kappa_{s}=3$, this sub-matrix becomes singular with all elements zero.

Circulant Sub-Matrix: The circulant block's eigenvalues are identified by the Discrete Fourier Transform (DFT) of a column (a well-known property of circulant matrices, see [12]).

$$
\begin{gathered}
\text { Upper Block E-values }=\mathcal{D} \mathcal{F} \mathcal{T}\left(\left[\begin{array}{llll}
2 \kappa_{s} & 6 & \ldots & 6
\end{array}\right]\right) \\
\quad=\left[\begin{array}{llll}
2 \kappa_{s}+6(N-1) & 2 \kappa_{s}-6 & \ldots & 2 \kappa_{s}-6
\end{array}\right]
\end{gathered}
$$

The first term $2 \kappa_{s}+6(N-1)$ is always positive and can be shown to correspond to the radial direction eigenvector (related through the transformation matrix $\mathbb{P}$ ). The signs of the remaining eigenvalues will depend on the value of $\kappa_{s}$. For:

$\boldsymbol{\kappa}_{s}<3$ : Here, $2 \kappa_{s}-6<0$. So, this block has one positive eigenvalue, and the remaining $N-1$ eigenvalues being negative.

$\boldsymbol{\kappa}_{s}>\mathbf{3}$ : Here, the sub-block has all $N$ eigenvalues being positive.

In any case, whenever $\kappa_{s} \neq 3, \widehat{\mathbb{M}}$ has mixed eigenvalues with the diagonal sub-matrix and circulant sub-matrix always containing eigenvalues of opposing signs.

$H \in \mathcal{C}_{n+1}$ (all non-zero) In this case, we have the entire matrix $\widehat{\mathbb{M}}$ being circulant, with eigenvalues of $2 \kappa_{s}+6(N-1)$ and $2 \kappa_{s}-6$. For $\kappa_{s}<3$, this is corresponds to $H$ being a saddle point. However, for $\kappa_{s}>3$, we have that all $H$ in this class are minima (with maximal ISI!).

\begin{tabular}{|l|c||c|c|c||l|}
\hline Class & \multicolumn{1}{|c||}{$\left|\mathcal{C}_{i}\right|$} & $\begin{array}{c}\text { \# pos. } \\
\text { eigvals }\end{array}$ & $\begin{array}{l}\# \text { neg. } \\
\text { eigvals }\end{array}$ & type & Comment \\
\hline$H \in \mathcal{C}_{0}$ & 1 & 0 & $n+1$ & max & Origin \\
\hline$H \in \mathcal{C}_{1}$ & $2(n+1)$ & $n+1$ & 0 & min & No ISI \\
\hline$H \in \mathcal{C}_{N}$ & $2^{N}\left(\begin{array}{c}n+1 \\
N\end{array}\right)$ & $n-N$ & $N+1$ & saddle & $2 \leq N \leq n$ \\
\hline$H \in \mathcal{C}_{n+1}$ & $2^{n+1}$ & 1 & $n$ & saddle & Max ISI \\
\hline
\end{tabular}

Table 1. Stationary Points, Platykurtic Source

\begin{tabular}{|l|c||c|c|c||l|}
\hline Class & $\left|\mathcal{C}_{i}\right|$ & $\begin{array}{l}\text { \# pos. } \\
\text { eigvals }\end{array}$ & $\begin{array}{l}\# \text { neg. } \\
\text { eigvals }\end{array}$ & type & Comment \\
\hline$H \in \mathcal{C}_{0}$ & 1 & 0 & $n+1$ & max & Origin \\
\hline$H \in \mathcal{C}_{1}$ & $2(n+1)$ & 1 & $n$ & saddle & No ISI \\
\hline$H \in \mathcal{C}_{N}$ & $2^{N}\left(\begin{array}{c}n+1 \\
N\end{array}\right)$ & $N+1$ & $n-N$ & saddle & $2 \leq N \leq n$ \\
\hline$H \in \mathcal{C}_{n+1}$ & $2^{n+1}$ & $n+1$ & 0 & min & Max ISI \\
\hline
\end{tabular}

\section{Table 2. Stationary Points, Leptokurtic Source}

\subsection{Summary of Results}

A summary of the stationary point stability analysis is found in Tables 1 and 2 .

Platykurtic: Only the solutions $H \in \mathcal{C}_{1}$ are stable points of the FSE-CMA error surface under an iid source with $\kappa_{s}<3$. As any $H \in \mathcal{C}_{1}$ is a perfect equalization setting we have that CMA is globally convergent to a perfect equalization setting in this case, duplicating the results of [4] [9].

Leptokurtic: However, in cases with $\kappa_{s}>3$, CMA is globally convergent to settings in which all taps have equal, nonzero values. Such parametrizations correspond to maximal ISI settings, providing disastrous equalization performance.

Meso-Kurtic: A Gaussian source is known to cause illbehavior of CMA and other blind equalization schemes [1] and we note that a Gaussian source distribution has $\kappa_{s}=3$. Our work here has shown that in the case of $\kappa_{s}=3 \mathrm{CMA}$ will adapt to any parametrization on the sphere which, in general, does not provide adequate equalization. However, the class of sources for which this behavior occurs is much broader than the oft-cited Gaussian class. There are many other distributions which may attain $\kappa_{s}=3$ (e.g. let $s_{i}= \pm 1$ with probability $p( \pm 1)=\frac{79-\sqrt{97}}{90}, s_{i}= \pm 4$ with probability $p( \pm 4)=\frac{11+\sqrt{97}}{90}$ ) which have this extreme effect on CMA. We propose a name of meso-kurtic to denote them. The point being that even non-Gaussian source distributions may cause problems with blind equalizers. In light of the potential for misinterpretation, it is worth pointing out again that we are referring to the source distribution, not the received signal distributions. This distinction is important since often the received distribution looks Gaussian after a non-Gaussian source signal is distorted through a channel with a large amount of ISI.

\subsection{Error Surface Curvature and Convergence}

The vast number of saddle points (on the order of $3^{n+1}$ ) might lead to slow convergence. This concern is heightened in cases where the source kurtosis increases towards 3. From (16) and (18) it is seen that as $\kappa_{s}$ approaches 3, all but one (the one in the a radial direction) eigenvalues of all stationary points approaches zero. The neighborhood 
of all saddle points becomes "flatter". This warns of prolonged convergence times. This should be especially noted in cases where CMA equalizers are used in conjunction with constellation shaping which increases the Gaussianity of the source (e.g. the v.34 modem standard). In [7] it is shown that optimal shaping for a large two-dimensional constellation is close to a Gaussian distribution. So, improved source coding may hinder blind equalization ability and the tradeoff should be considered in the design of a communication system. In [13] this issue is considered mostly for non-CMA algorithms.

Example A: An example of convergence rate decrease due to curvature reduction resulting from source kurtosis increasing towards $\kappa_{s}=3$ is demonstrated in Figure 2. Here the ISI trajectories are plotted for a variety of source kurtosis values. The equalizers are initialized with a center tap initialization $\left(d=\left[\begin{array}{lllll}0 & 0 & 1 & 0 & 0\end{array}\right]^{t}\right)$ and the source distributions have $1.76<\kappa_{s}<5.04$. ISI is reduced by CMA-FSE if $\kappa_{s}<3$, while ISI is enhanced when $\kappa_{s}>3$, agreeing with results. Furthermore, for $\kappa_{s}<3$, the larger the source kurtosis, the slower the convergence as all but one of the eigenvectors of the Hessian evaluated at all of the stationary points diminish as $\kappa_{s} \rightarrow 3$ from below.

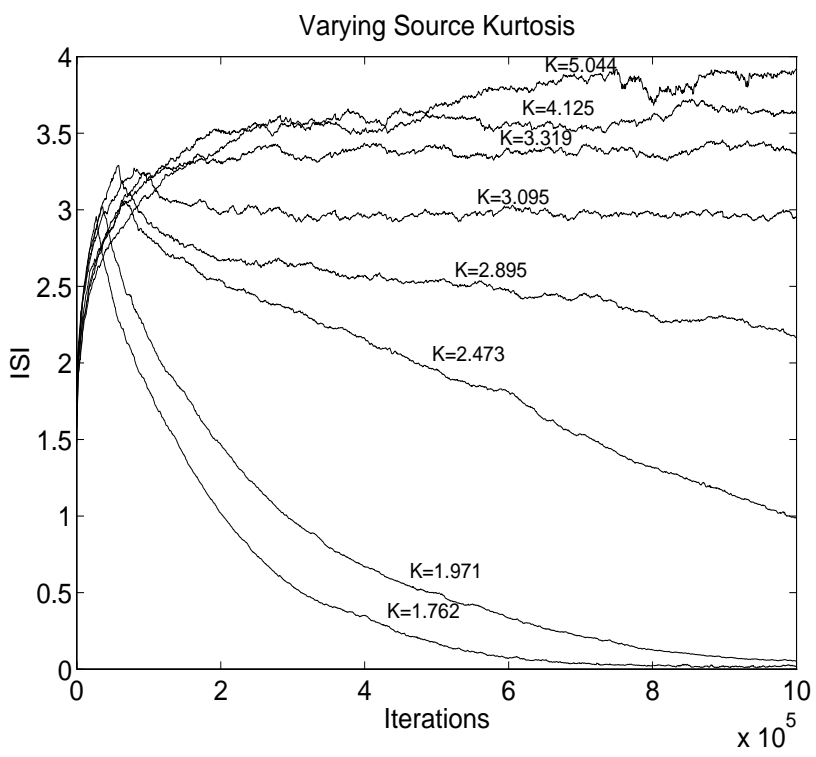

Figure 2. ISI trajectories for various $\kappa_{s}$

Example B: Next, we view the effect of kurtosis on convergence time. Here the equalizer is initialized so that $\left.H=\left[\begin{array}{lllll}1 & 1 & 1 & 1 & 1\end{array}\right]^{t}\right)$. Using the same channel and stepsize $\mu$ as in examples $A$, the number of iterations needed to reduce ISI to an open-eye setting (which represents the earliest time for a switch to decision directed is guaranteed to produce no decision errors (in the absence of channel noise) is computed for a variety of source kurtosis values $\left(\kappa_{s}<3\right)$.

Figure 3 reflects the dependence of convergence time on source kurtosis and highlights the necessity of avoiding a source kurtosis too close to $\kappa_{s}=3$. Thus, we have an apparent tradeoff between source coding gains (driving $\kappa_{s} \rightarrow 3$ ) and blind equalizer convergence (preferring $\kappa_{s}$ away from value 3$)$.

\section{REFERENCES}

[1] A. Benveniste, M. Goursat, G. Ruget, "Robust Identification of a Nonminimum Phase System: Blind Adjustment of a Linear Equalizer in Data Communications,"

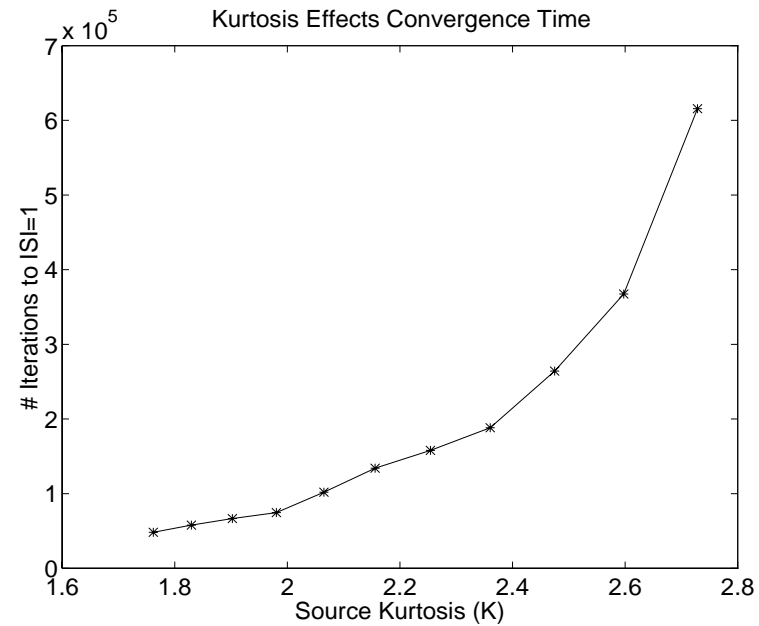

Figure 3. Eye-opening Time vs. Kurtosis

IEEE Trans. on Automat. Contr., vol. AC-25, no. 3, Jun. 1980.

[2] C. K. Chan, J. J. Shynk, "Stationary Points of the Constant Modulus Algorithm for Real Gaussian Signals ," IEEE Trans. Acoust. Speech Signal Process., vol. 38, Dec. 1990.

[3] D.L. Donoho, "On Minimum Entropy Deconvolution," Applied Time Series Analysis, D.F. Findley, Ed., New York: Academic Press, 1981.

[4] I. Fijalkow, C. E. Manlove, C. R. Johnson, Jr., "Adaptive Fractionally Spaced Blind CMA Equalization," Submitted to IEEE trans. on Signal Processing, 1994.

[5] D.N. Godard, "Self-Recovering Equalization and Carrier Tracking in Two-Dimensional Data Communication Systems," IEEE Trans. on Commun., vol. COM28, No. 11, Nov. 1980.

[6] C. R. Johnson, Jr., B. D. O. Anderson, "Godard Blind Equalizer Error Surface Characteristics," Int'l Journal of Adaptive Control and Signal Processing, in press.

[7] R. Laroia, N. Farvardin, S. A. Tretter, "On Optimal Shaping of Multidimensional Constellations," IEEE Trans. on Info. Theory, vol. 40, No. 4, July 1994.

[8] J. P. LeBlanc, I. Fijalkow, B. Huber, C. R. Johnson, Jr., "Fractionally Spaced CMA Equalizers Under Periodic and Correlated Inputs," Proc. of IEEE Conf. Acoust., Speech, Signal Processing, May 1995.

[9] Y. Li, Z. Ding, "Convergence Analysis of Finite Length Blind Adaptive Equalizers," IEEE Trans. on Signal Process., Sept. 1995.

[10] L. Tong, G. Xu, T. Kailath, "Blind Identification and Equalization Based on Second-Order Statistics: A Time Domain Approach," IEEE Trans. on Info. Theory, Mar. 1994.

[11] J.R. Treichler, M. G. Agee, "A New Approach to Multipath Correction of Constant Modulus Signals," IEEE Trans. on Acoustics, Speech, and Signal Processing, April 1983.

[12] A. S. Willsky, Digital Signal Processing and Control and Estimation Theory: Points of Tangency, Areas of Intersection, and Parallel Directions, Cambridge, Massachusetts: MIT Press, 1979. 
[13] E. Zervas, J. G. Proakis, V. Eyuboglu, "Effects of Constellation Shaping on Blind Equalization," SPIE, 1991. 